\title{
Myocardial fibrosis in arterial hypertension
}

\author{
A. González ${ }^{1}$ B. López ${ }^{1}$ and J. Díez ${ }^{1,2}$ \\ ${ }^{1}$ Division of Cardiovascular Pathophysiology, School of Medicine, and ${ }^{2}$ Department of Cardiology and Cardiovascular \\ Surgery, University Clinic, University of Navarra, Pamplona, Spain
}

It is now accepted that, in addition to left ventricular hypertrophy, hypertensive heart disease is characterized by alterations in myocardial structure, leading to loss of tissue homogeneity and pathological remodelling. It is time to recognize that, in hypertensive heart disease, it is not only the quantity but also the quality of the myocardium that is responsible for adverse cardiovascular events. The data reviewed here indicate that, in patients with hypertensive heart disease, myocardial fibrosis predisposes to an enhanced risk for diastolic and/or systolic ventricular dysfunction, symptomatic heart failure, ischaemic heart disease and arrhythmias.

(Eur Heart J Supplements 2002; 4 (Suppl D): D18-D22)

(C) 2002 The European Society of Cardiology

Key Words: Fibrosis, heart failure, hypertension, myocardium, remodelling, reparation.

\section{Introduction}

A growing body of evidence indicates that myocardial fibrosis is among the key pathological features of myocardial remodelling in hypertensive heart disease. Indeed, studies performed in post-mortem human hearts ${ }^{[1]}$ and human endomyocardial biopsies ${ }^{[2]}$ show that myocardial collagen volume fraction (a morphometric measure of the amount of tissue collagen) is consistently greater in patients with hypertensive heart disease than in normotensive control individuals. Furthermore, in patients with hypertensive heart disease, immunohistochemical analyses show exaggerated accumulation of fibrillar collagen types I and III within the myocardial interstitium and surrounding intra-mural. coronary arteries and arterioles.

The present brief review focuses on the pathophysiology of hypertensive myocardial fibrosis and its detrimental impact on cardiac function. It also discusses the noninvasive diagnostic tools that are currently under evaluation to detect myocardial fibrosis, and the evidence for its reparation in patients with hypertensive heart disease.

Correspondence: Dr Javier Díez, División de Fisiopatología Cardiovascular, Facultad de Medicina, C/ Irunlarrea s/n, 31080 Pamplona, Spain.

\section{Multifactorial origin}

It has been proposed that the excess of myocardial collagen seen in hypertension is the result of both increased collagen synthesis and unchanged or decreased collagen degradation ${ }^{[3]}$. This hypothesis is supported by recent experimental findings that show upregulation of the procollagen type I gene[4] and diminished collagenase activity $\left.{ }^{5}\right]$ in the hypertrophied and fibrotic left ventricles of adult spontaneously hypertensive rats. Various factors (haemodynamic, humoral and genetic) may account for this disequilibrium (Fig. 1).

\section{Haemodynamic factors}

In-vivo experiments have shown that chronic pressure overload stimulates both pro-collagen gene expression and collagen protein synthesis, leading to excessive collagen deposition and fibrosis[6]. In addition, in-vitro studies have shown that pro-collagen type I synthesis is stimulated in cardiac fibroblasts subjected to cyclic mechanical load[6]. Thus, haemodynamic overload of the left ventricle due to systemic hypertension may play a role in myocardial fibrosis. 


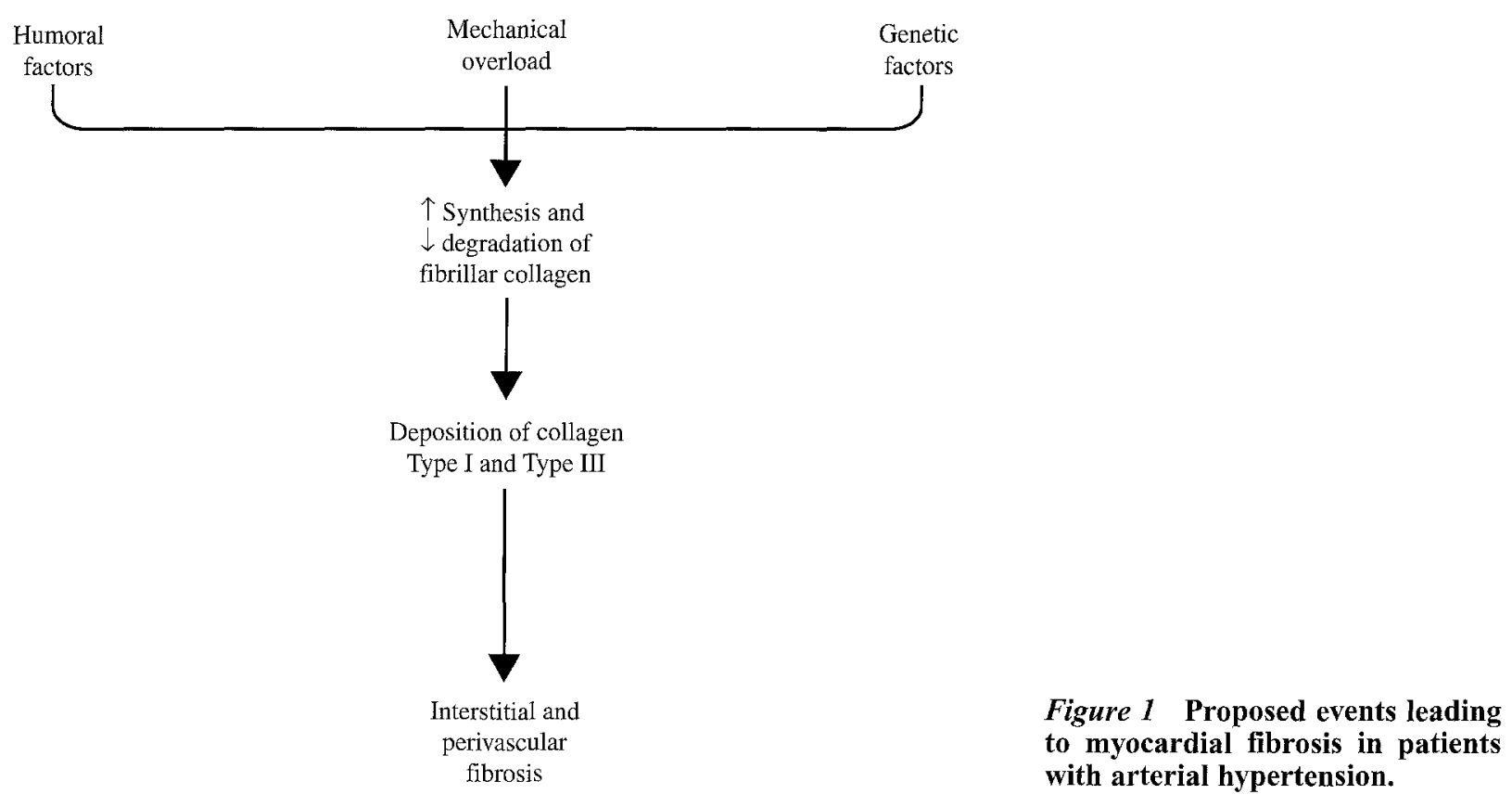

\section{Humoral factors}

In addition to haemodynamic factors, non-haemodynamic factors may also contribute to myocardial fibrosis in human hypertension, as suggested by two types of finding. First, post-mortem studies conducted in human hypertensive heart disease have identified myocardial fibrosis not only in the left ventricle but also in the right ventricle ${ }^{[7]}$ and the interventricular septum ${ }^{[8]}$. Second, recent studies have shown that antihypertensive agents can cause regression of biopsyproven myocardial fibrosis in hypertensive patients, independent of antihypertensive efficacy $[9,10]$.

Thus, the current view is that myocardial fibrosis may result from the loss of reciprocal regulation that normally exists between molecules that stimulate and inhibit fibrillar collagen turnover ${ }^{[3]}$. Stimulatory molecules include angiotensin II, aldosterone, transforming growth factor-beta and endothelin; inhibitory molecules include bradykinin, prostaglandins, nitric oxide and natriuretic peptides. An excess of stimulators, absolute (due to over-production) or relative (due to a deficit in inhibitor formation), can promote fibrosis.

\section{Genetic factors}

Preliminary data suggest that hypertensive myocardial fibrosis may also be under genetic control. In a recent study[11], we investigated whether $\mathrm{A}^{1166} \mathrm{C}$ angiotensin II type 1 receptor $\left(A T_{1}\right)$ polymorphism influences the ability of losartan to inhibit synthesis of collagen type I and to bring about regression of myocardial fibrosis in patients with hypertensive heart disease. Patients were genotyped for the $\mathrm{A}^{1166} \mathrm{C} \mathrm{AT}_{1}$ receptor polymorphism and divided into two subgroups: AA and $\mathrm{AC} / \mathrm{CC}$. Baseline demographic and haemodynamic parameters were comparable in the two groups of patients. However, collagen volume fraction was higher in AA patients than in $\mathrm{AC} / \mathrm{CC}$ patients $(P<0 \cdot 05)$.

\section{Detrimental impact}

Myocardial fibrosis predisposes to heart failure (Fig. 2), diminished coronary reserve and ventricular arrhythmias, and may thereby increase the risk for adverse cardiovascular events in patients with hypertensive heart disease.

Various clinical and experimental studies have demonstrated the importance of fibrosis in causing tissue stiffness. As reviewed elsewhere[12], these studies addressed the following: the presence of fibrosis in the hypertrophied ventricle with abnormal stiffness; the importance of fibrosis in causing abnormal myocardial stiffness, with or without cardiomyocyte hypertrophy; whether preventing fibrosis would preserve normal tissue stiffness; and whether regression of fibrosis would normalize tissue stiffness in hypertrophied or non-hypertrophied ventricles. As a result of those studies, the following broad statement can be made[13]. A two- to threefold increase in collagen volume fraction increases diastolic stiffness (or diastolic dysfunction), whereas systolic stiffness is preserved. A fourfold or greater rise in collagen volume fraction is associated with an additional rise in diastolic stiffness and an increase in systolic stiffness that may facilitate systolic dysfunction.

This statement is supported by several clinical findings. Sugihara et al.[14] and Ohsato et al. ${ }^{[15]}$ reported that, in hypertensive patients, collagen volume fraction was the most significant factor related to diastolic dysfunction, as assessed using Doppler echocardiography. Furthermore, in hypertensive patients, López et al.[10] found an inverse correlation between collagen volume fraction and Doppler 


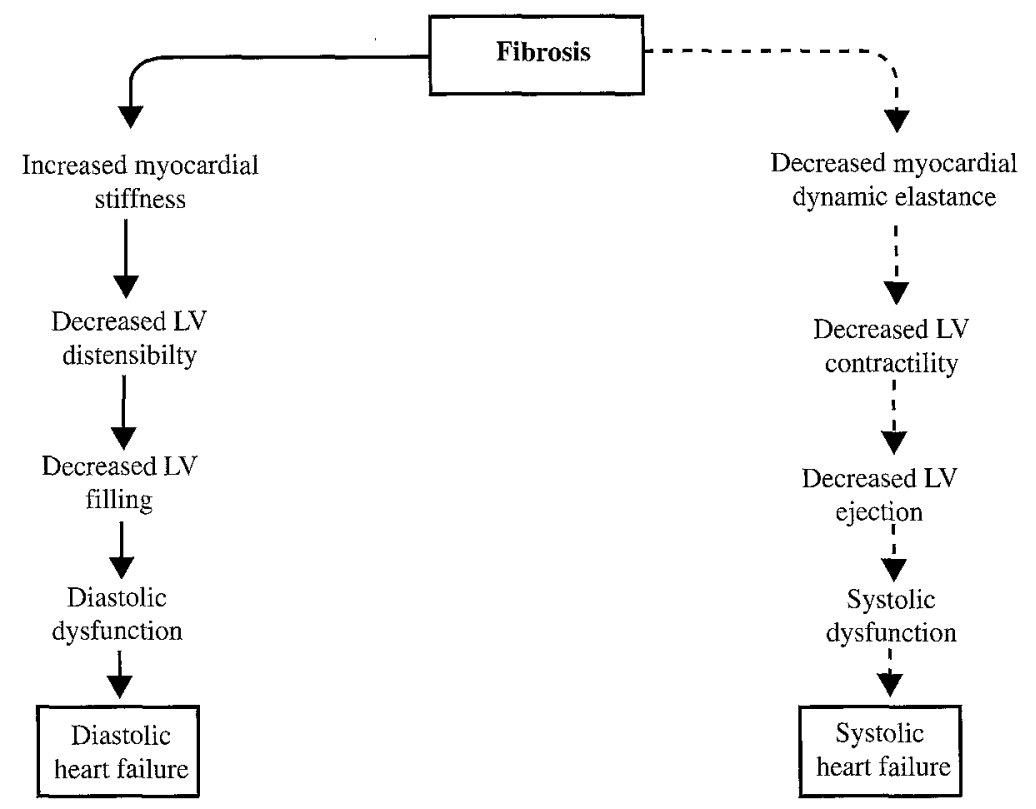

Figure 2 Proposed detrimental consequences of myocardial fibrosis on left ventricular $(\mathrm{LV})$ function in patients with arterial hypertension. mitral A wave deceleration time (an index of left ventricular distensibility).

Recently, Brilla et al..$^{9]}$ reported that, in patients with essential hypertension, chronic treatment with the angiotensin-converting enzyme (ACE) inhibitor lisinopril reduced collagen volume fraction and improved left ventricular diastolic function. Those changes were independent of regression in left ventricular hypertophy.

An inverse relationship has been found between left ventricular ejection fraction and collagen volume fraction in hypertensive patients ${ }^{[16]}$. Furthermore, that study found that systolic function was preserved when moderate fibrosis (collagen volume fraction 5-10\%) was present, but declined when fibrosis became more severe.

Additional epidemiological and clinical data strengthen the potential relevance of these findings. Diastolic heart failure accounts for $30-50 \%$ of congestive heart failure in clinical practice, and is mainly caused by hypertensive heart disease [17]. Diastolic heart failure has a significant effect on mortality (5-year mortality rate of 25-35\%) and morbidity (1-year readmission rate of $50 \%)^{[18]}$. Exercise-induced systolic dysfunction in hypertensive patients with left ventricular hypertrophy has been found to arise predominantly from impaired diastolic filling, leading to insufficient augmentation of end-diastolic filling during exercise to maintain systolic function ${ }^{[19]}$. Recent findings in patients with hypertensive pulmonary oedema and normal left ventricular ejection fraction after treatment[20] indicate that pulmonary congestion was probably due to isolated, transient diastolic dysfunction, with increases in both left ventricular end-diastolic pressure and left atrial pressure.

Schwartzkopff et al.[21] demonstrated that, in hypertensive patients with reduced coronary flow reserve, total and peri-vascular collagen volume fraction is correlated with the increased minimal coronary resistance. Furthermore, the same group showed that, in hypertensive patients, long-term therapy with the $\mathrm{ACE}$ inhibitor perindopril induces a decrease in coronary resistance and improvement in coronary reserve, associated with significant regression of peri-arteriolar fibrosis ${ }^{[22]}$

Finally, McLenachan and Dargie [23] analysed possible correlates of left ventricular arrhythmias in patients with hypertensive heart disease. They found that patients with arrhythmias had higher left ventricular mass and collagen volume fraction than did patients without arrhythmias. Ejection fraction and the frequency of coronary vessels with significant ( $>50 \%)$ stenosis were similar in the two groups of patients. Thus, the high incidence of arrhythmias in patients with hypertensive heart disease cannot entirely be attributed to coexistent coronary artery disease or to left ventricular dysfunction. On the contrary, it may be related to fibrosis and the adaptive phenotypic changes in membrane proteins associated with cardiomyocyte hypertrophy.

\section{Non-invasive detection}

Because of the adverse effects of myocardial fibrosis in patients with hypertensive heart disease, determining the extent of collagen accumulation in tissue may be helpful in predicting clinical outcome. It may also assist in designing strategies to prevent or even reverse the development of fibrosis.

Although endomyocardial biopsy is a simple and safe outpatient procedure, it is nevertheless an invasive methodology with obvious limitations for widescale application. Non-invasive measures of cardiac fibrosis (i.e. ultrasound tissue characterization and biochemical monitoring of collagen turnover) are therefore desirable (Fig. 3).

Studies conducted in humans with various disorders involving myocardial fibrosis ${ }^{[24,25]}$ have shown a promising correlation between echo-reflectivity and histologically assessed collagen content. Moreover, Ciulla et al.[26] demonstrated a direct correlation between collagen volume fraction and echo amplitude in patients with hypertensive 


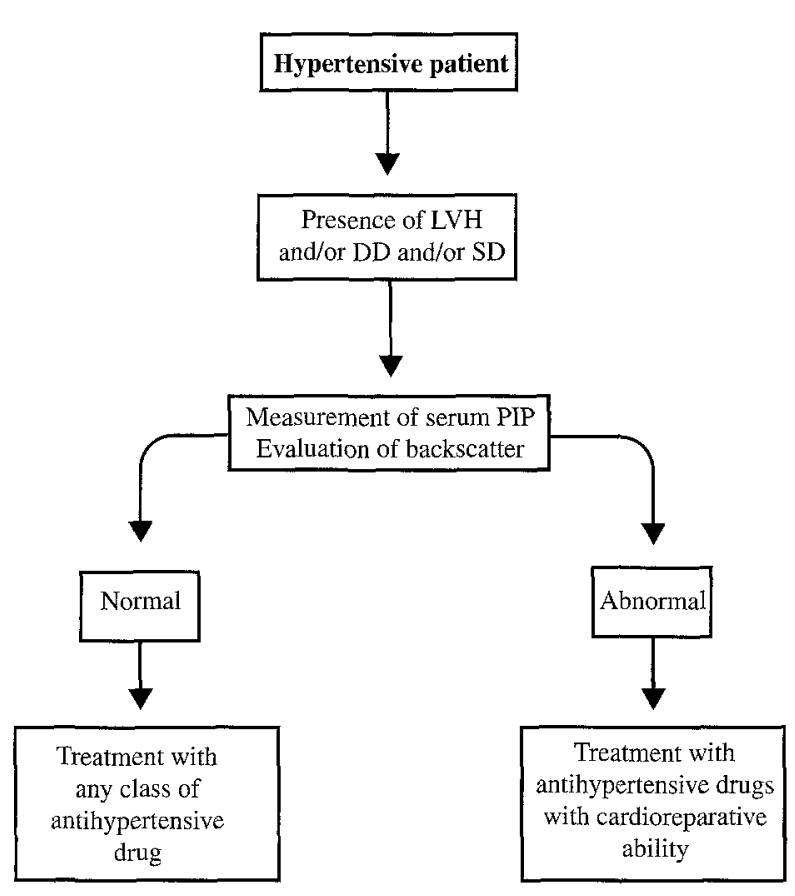

Figure 3 Proposed algorithm for detection and management of myocardial fibrosis in patients with arterial hypertension. $\mathrm{DD}=$ diastolic dysfunction; $\mathrm{LVH}=$ left ventricular hypertrophy; $\mathrm{PIP}=$ pro-collagen type I carboxyl-terminal pro-peptide; $\mathrm{SD}=$ systolic dysfunction.

heart disease, suggesting that collagen content is the major determinant of regional echo intensity. Other studies ${ }^{[27]}$ have also demonstrated alterations in the pattern of myocardial reflectivity in patients with hypertensive heart disease, especially in those with severe left ventricular hypertrophy.

Radioimmunoassays of various serological markers of collagen type I and type III turnover have also shown promise in clinical studies in patients with arterial hypertension. Pro-collagen type I carboxyl-terminal propeptide (PIP) is a marker of collagen type I synthesis. In several studies, we found that serum concentrations of PIP were significantly higher in patients with hypertensive heart disease than in normotensive control individuals $[2,10,28]$. We also found that serum PIP concentrations correlated directly with collagen volume fraction in patients with hypertensive heart disease ${ }^{2}$.

Using receiver operating characteristic curves, we calculated that a cutoff of $127 \mu \mathrm{g} \mathrm{.} 1^{-1}$ for PIP provided $78 \%$ specificity and $75 \%$ sensitivity for predicting severe myocardial fibrosis, with a relative risk of 4.80 (95\% confidence interval 1/19-10.30). Furthermore, when a cutoff of $126 \mu \mathrm{g} \mathrm{.} 1^{-1}$ for PIP and $205 \mathrm{~ms}$ for deceleration time were combined, specificity for predicting severe myocardial fibrosis was maintained, and sensitivity rose to $100 \%$ with a relative risk of $8 \cdot 50(95 \%$ confidence interval $2 \cdot 31-31 \cdot 25)$. In another study[10], we observed a strong association between treatment-induced changes in collagen volume fraction and treatment-induced changes in serum PIP in patients with hypertensive heart disease.

\section{Management: potential for cardioreparation}

Reparation of pathological remodelling in hypertensive heart disease focuses on regression of fibrosis and reduction in the associated risk for cardiovascular events (Fig. 3) ${ }^{[29]}$. A cardioreparative agent should counteract the imbalance between inhibitors and stimulators of turnover of collagen types I and III. Antihypertensive agents that appear to meet this requirement include $\mathrm{ACE}$ inhibitors, $\mathrm{AT}_{1}$ antagonists, aldosterone antagonists and vasopeptidase inhibitors.

The cardioreparative concept has been proved clinically in three recent, relatively small, prospective trials in patients with biopsy-proved myocardial fibrosis. Schwartzkopff et al. ${ }^{[22]}$ treated 14 patients with essential hypertension for 12 months with the ACE inhibitor perindopril. Treatment was associated with structural repair of coronary arterioles, characterized mainly by the regression of peri-arteriolar fibrosis, and was associated with a marked improvement in coronary reserve. Brilla et al. ${ }^{[9]}$ randomized 35 patients with hypertensive heart disease to receive either the ACE inhibitor lisinopril or the diuretic hydrochlorothiazide for 6 months. Only patients randomized to lisinopril had a significant reduction in collagen volume fraction, which was associated with improvement in diastolic dysfunction. Finally, López et al.[10] studied 37 patients with hypertensive heart disease. After randomization, 21 patients were assigned to receive the $\mathrm{AT}_{1}$ receptor antagonist losartan, and 16 to the calcium channel blocker amlodipine for 12 months. In losartan-treated patients, collagen volume fraction decreased and left ventricular distensibility increased significantly. In contrast, those parameters remained unchanged in amlodipine-treated patients.

\section{Conclusion}

Myocardial fibrosis is a key histological constituent of myocardial remodelling in hypertensive heart disease. Hypertensive myocardial fibrosis has been shown to promote abnormalities of cardiac function that may adversely affect the clinical outcome of hypertensive patients. Development of non-invasive tools for monitoring myocardial fibrosis and pharmacological strategies to promote its regression could therefore be of particular relevance in the clinical management of patients with hypertensive heart disease.

\section{References}

[1] Rossi MA. Pathologic fibrosis and connective tissue matrix in left ventricular hypertrophy due to chronic arterial hypertension in humans. J Hypertens 1998; 16: 1031-41.

[2] Querejeta R, Varo N, López B et al. Serum carboxy-terminal propeptide of procollagen type I is a marker of myocardial fibrosis in hypertensive heart disease. Circulation 2000; 101: 1729-35.

[3] Weber KT. Fibrosis and hypertensive heart disease. Curr Opin Cardiol 2000; 15: 264-72. 
[4] Varo N, Etayo JC, Zalba G et al. Losartan inhibits the posttranscriptional synthesis of collagen type I and reverses left ventricular fibrosis in spontaneously hypertensive rats. J Hypertens 1999; 17: 107-14.

[5] Varo N, Iraburu MJ, Varela $\mathrm{M}$ et al. Chronic $\mathrm{AT}_{1}$ blockade stimulates extracellular collagen type I degradation and reverses myocardial fibrosis in spontaneously hypertensive rats. Hypertension 2000; 35: 1197-202.

[6] Bishop JE, Lindahl G. Regulation of cardiovascular collagen synthesis by mechanical load. Cardiovase Res 1999; 42: 27-44.

[7] Olivetti G, Melissari M, Balbi T et al. Myocyte cellular hypertrophy is responsible for ventricular remodelling in the hypertrophied heart of middle aged individuals in the absence of cardiac failure. Cardiovasc Res 1994; 28: 1199-208.

[8] Pearlman ES, Weber KT, Janicki JS et al. Muscle fiber orientation and connective tissue content in the hypertrophied human heart. Lab Invest 1982; 46: 158-64.

[9] Brilla CG, Funck RC, Rupp H. Lisinopril-mediated regression of myocardial fibrosis in patients with hypertensive heart disease. Circulation 2000; 102: 1388-93.

[10] López B, Querejeta R. Varo N et al. Usefulness of serum carboxyterminal propeptide of procollagen type I in assessment of the cardioreparative ability of antihypertensive treatment in hypertensive patients. Circulation 2001; 104: 286-91.

[11] López B, Querejeta R, Varo $\mathrm{N}$ et al. Chronic $\mathrm{AT}_{1}$ blockade decreases collagen type I synthesis and reverses myocardial fibrosis in hypertensive patients [abstract]. JRAAS 2001;2:52.

[12] Weber KT, Brilla CG, Janicki JS. Myocardial fibrosis: functional significance and regulatory factors. Cardiovase Res 1993; 27 : $341-8$.

[13] Weber KT. Cardiac interstitium. In: Poole-Wilson PA, Colucci WS, Massie BM, Chatterjee K, Coats AJS, eds. Heart Failure. Scientific Principles and Clinical Practice. New York, Churchill Livingstone, 1997: 13-31.

[14] Sugihara N, Genda A, Shimizu M et al. Diastolic dysfunction and its relation to myocardial fibrosis in essential hypertension. J Cardiol 1988; 18: 353-61.

[15] Ohsato K, Shimizu M, Sugihara N et al. Histopathological factors related to diastolic function in myocardial hypertrophy. Jpn Circ J 1992; 56: 325-33.

[16] McLenachan JM, Dargie HJ. Ventricular arrhythmias in hypertensive left ventricular hypertrophy. Relationship to coronary artery disease, left ventricular dysfunction, and myocardial fibrosis. Am J Hypertens 1990; 3: 735-40.
[17] Vasan RS, Larson MG, Benjamin EJ et al. Congestive heart failure in subjects with normal versus reduced left ventricular ejection fraction: prevalence and mortality in a population-based cohort. J Am Coll Cardiol 1999; 33: 1948-55.

[18] Zile MR, Nappi J. Diastolic heart failure. Curr Treat Options Cardiovasc Med 2000; 5: 439-50.

[19] Cuocolo A, Sax FL, Brush JE et al. Left ventricular hypertrophy and impaired diastolic filling in essential hypertension. Diastolic mechanisms for systolic dysfunction during exercise. Circulation 1990; 81: 978-86.

[20] Ghandi SK, Powers JC, Nomeir A-M et al. The pathogenesis of acute pulmonary edema associated with hypertension. $\mathrm{N}$ Engl J Med 2001; 344: 17-22.

[21] Schwartzkopff B, Motz W, Frenzel H et al. Structural and functional alterations of the intramyocardial coronary arterioles in patients with arterial hypertension. Circulation 1993; 88: 993-1003.

[22] Schwartzkopff B, Brehm M, Mundhenke M et al. Repair of coronary arterioles after treatment with perindopril in hypertensive heart disease. Hypertension 2000; 36: 220-5.

[23] McLenachan JM, Dargie HJ. Ventricular arrhythmias in hypertensive left ventricular hypertrophy. Relationship to coronary artery disease, left ventricular dysfunction, and myocardial fibrosis. Am J Hypertens 1990; 3: 735-40.

[24] O'Donnell M, Mimbs JW, Miller JG. The relationship between collagen and ultrasonic backscatter in myocardial tissue. J Acoust Soc Am 1981; 69: 580-8.

[25] Picano E, Pelosi G, Marzilli M et al. In vivo quantitative ultrasonic evaluation of myocardial fibrosis in humans. Circulation 1990; 81: 58-64.

[26] Ciulla M, Paliotti R, Hess DB et al. Echocardiographic patterns of myocardial fibrosis in hypertensive patients: endomyocardial biopsy versus ultrasonic tissue characterization. J Am Soc Echocardiogr 1997; 10: 657-64.

[27] Lucarini AR, Talarico L, Di Bello V et al. Increased myocardial ultrasonic reflectivity is associated with extreme hypertensive left ventricular hypertrophy: a tissue characterization study in humans. Am J Hypertens 1998; 11: 1442-9.

[28] Laviades C, Varo N, Diez J. Transforming growth factor- $\beta$ in hypertensives with cardiorenal damage. Hypertension 2000; 36: $517-22$.

[29] Weber KT. Cardioreparation in hypertensive heart disease. Hypertension 2001; 38: 588-91. 